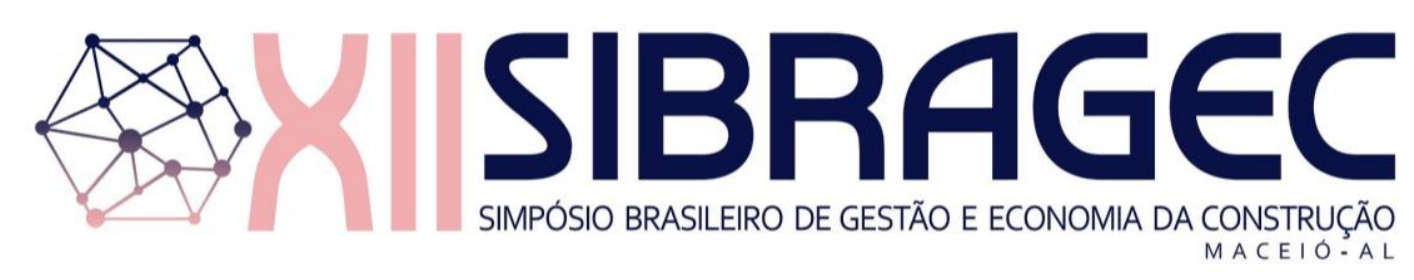

\title{
AVALIAÇÃO DO COMPORTAMENTO ENXUTO DE FISCAIS DE OBRAS $^{1}$
}

\section{OLIVEIRA, Kaio Pimentel Rego de (1); MONTEIRO, Camila de Oliveira (2); SANTOS, Débora de Gois (3)}

(1) Universidade Federal de Sergipe, kaiopimentel1@gmail.com, (2) Universidade Federal de Sergipe, camiladeomonteiro@gmail.com, (3) Universidade Federal de Sergipe,

deboragois@yahoo.com.br

\begin{abstract}
RESUMO
As obras públicas possuem notável representatividade de investimentos no Brasil. Os últimos dados do IBGE apontam que as Entidades Públicas foram responsáveis por 30,7\% do valor bruto de produção da Construção Civil. Visto a notoriedade dessas obras e seu papel na sociedade, é necessário adotar mecanismos de gestão que melhorem a eficiência dos empreendimentos e do uso dos recursos. Para tanto, autores apontam a importância de avaliar o comportamento enxuto das instituições, fornecendo embasamento para a tomada de decisão acerca da implantação de princípios modernos de gestão da produção. Observou-se, porém, lacuna de conhecimento na avaliação deste cenário com fiscais de obras públicas. Nesse contexto, objetiva-se fornecer um diagnóstico identificando conceitos enxutos que são aplicados ou pouco abordados por fiscais de obras públicas. O método abrange uma pesquisa exploratória e descritiva, envolvendo aplicação de questionário, identificando níveis de conhecimento enxuto dos fiscais de um órgão público, por estado brasileiro. Como principal resultado, observou-se um maior domínio dos fiscais nas áreas de comunicação e um menor domínio na área de definição do trabalho. Como contribuição, este trabalho fornece base para tomadas de decisão de órgãos públicos, visando intensificar a capacitação nas áreas de menor domínio e trazer ganhos mais expressivos à Administração Pública.
\end{abstract}

Palavras-chave: Construção Enxuta, Obras Públicas, Comportamento.

\begin{abstract}
Public works have a notable representativeness of investments in Brazil. The latest data from IBGE show that Public Entities were responsible for $30.7 \%$ of the gross production value of Civil Construction. With the notoriety of these works and their role in society, it is necessary to adopt management mechanisms that improve the efficiency of the enterprises and the use of resources. Therefore, authors point out the importance of assessing lean behavior of institutions, providing basis for decision making about the implementation of modern production management principles. However, there was a knowledge gap in the assessment of this scenario with public works inspectors. In this context, the objective is to provide a diagnosis identifying lean concepts that are applied or poorly addressed by public works inspectors. The method is an exploratory and descriptive research, involving the application of a questionnaire, identifying levels of lean knowledge of the inspectors of a public agency, by Brazilian state. As a main result, there was a greater mastery of inspectors in the areas of communication and a lesser mastery in the area of work definition. As a contribution, this work provides the basis for agencies' decision making, aiming to intensify training in areas of lesser mastery and bring more significant gains to the Public Administration.
\end{abstract}

Keywords: Lean Construction, Public Works, Behavior.

\footnotetext{
${ }^{1}$ OLIVEIRA, K. P. R. de; MONTEIRO, C. de O.; SANTOS, D. de G. Avaliação do comportamento enxuto de fiscais de obras públicas. In: SIMPÓSIO BRASILEIRO DE GESTÃO E ECONOMIA DA CONSTRUÇÃO, 12., 2021, Maceió. Anais[...] Porto Alegre: ANTAC, 2021. p.1-9. Disponível em: https://eventos.antac.org.br/index.php/sibragec/article/view/456. Acesso em: 2 out. 2021.
} 


\section{INTRODUÇÃ̃o}

O Governo, dentre as suas diversas funções, deve proporcionar à sociedade melhorias de vida na esfera social. As obras são uma forma da Administração Pública para alcançar esse objetivo (Alvarenga, 2019) e possuem notável representatividade de investimentos no Brasil. De acordo com a última PAIC - Pesquisa Anual da Indústria da Construção, realizada em 2018, as Entidades Públicas foram responsáveis por 30,7\% do valor bruto de produção da Construção Civil (IBGE, 2018).

Para Corrêa e Shih (2019), as obras públicas devem oferecer uma infraestrutura adequada para trabalho e atendimento da comunidade, cabendo aos gestores fazer com que esse objetivo seja atingido de forma a minimizar os custos e maximizar os resultados. Para tanto, todos os envolvidos no processo de contratação e execução possuem grande responsabilidade para o sucesso de todas as etapas (BRANDSTETTER; RIBEIRO, 2019).

Assim, a Administração Pública deve adotar mecanismos de gestão nas práticas administrativas, visando melhorar a eficiência dos empreendimentos (CASOTTE, 2016). Diante disso, através da aplicação de seus conceitos, autores apontam a Construção Enxuta (Lean Construction) como uma filosofia gerencial que pode mitigar a ocorrência de desvios do planejamento inicial em obras públicas, sobretudo no que diz respeito aos prazos, através da redução do tempo de atravessamento dos processos (ANDÚJARMONTOYA et al., 2020; WEBER, 2020; BABALOLA et al., 2019).

Neste caminho, Bressiani et al. (2003) apresentam a importância de levantar e avaliar o comportamento enxuto das organizações, identificando conceitos que ainda são pouco abordados e dificuldades na aplicação da filosofia enxuta, fornecendo, assim, embasamento para uma melhor tomada de decisão acerca da implantação de princípios modernos de gestão da produção. Para tanto, os autores apresentam um questionário que levanta a presença do comportamento enxuto na vida e rotina do respondente, avaliando atitudes, comportamentos e opiniões (BRESSIANI et al., 2003). Mais tarde, no ano de 2009, estas questões foram publicadas na Coletânea Edificar Lean: Construindo com o Lean Management. (HEINECK et al., 2009).

Apesar da notoriedade das obras públicas no país, sua importância para a sociedade e a necessidade de propor mecanismos de gestão que otimizem o uso dos recursos e tragam um melhor desempenho aos empreendimentos, observou-se uma lacuna de conhecimento na avaliação do comportamento enxuto de fiscais de obras públicas. Nesse contexto, o presente trabalho objetiva fornecer um diagnóstico identificando os conceitos enxutos que são aplicados ou pouco abordados por fiscais deste tipo de obra. $\mathrm{O}$ universo de estudo são fiscais de obras públicas de um órgão público federal, com atuação a nível nacional.

\section{MÉTODO DE PESQUISA}

Para avaliar o conhecimento dos fiscais acerca da Construção Enxuta, foi aplicado um questionário estruturado, adaptado do original "Você tem comportamento Lean?", de Heineck et al. (2009), publicado na Coletânea Edificar Lean: Construindo com o Lean Management, cujo objetivo é testar o comportamento, atitudes e ações gerenciais enxutas do respondente.

As perguntas do questionário original foram adaptadas, sem perder a sua essência, para aplicação com os fiscais de obras públicas, cuja função abrange a inspeção e o controle técnico da obra ou serviço contratado, garantindo que a execução obedeça aos parâmetros exigidos no contrato.

Após a adaptação, o questionário foi submetido à avaliação do Comitê de Ética em Pesquisa (CAAE: 35793120.0.0000.5546). Após aprovação, foi lançado na plataforma 
on-line free Google Formulários ${ }^{\circledR}$ e, posteriormente, enviado por e-mail aos fiscais do órgão objeto do estudo de todas as Unidades Federativas do Brasil. O período de aplicação foi de 10 de novembro de 2020 a 10 de dezembro de 2020.

O questionário é composto por 5 grupos, divididos da seguinte maneira:

- Grupo 01: Definição do trabalho;

- Grupo 02: Medição do trabalho;

- Grupo 03: Comunicação e disseminação de informações;

- Grupo 04: Direção e motivação dos trabalhadores;

- Grupo 05: Uso de equipamentos.

De maneira geral, os grupos 01 e 05 avaliam a área de valor, ciclo, fluxo, desperdícios, padronização e tecnologia básica. Já os grupos 02, 03 e 04 avaliam a área de comunicações, motivação, melhoria das condições de trabalho, medição e melhoria da produção.

O questionário original possui 7 grupos. Porém, um grupo que avalia a área de programação dos trabalhos e outro grupo que avalia a relação com a cadeia produtiva, foram retirados na adaptação. Isso aconteceu porque os fiscais de obras públicas do órgão, dentro de suas funções, não programam os trabalhos executados, nem trabalham diretamente com a cadeia produtiva, apenas fiscalizando-os.

Os dados obtidos a partir da aplicação do questionário foram lançados em uma planilha eletrônica, tratados, divididos por UF respondente e foram determinados os percentuais de respostas SIM, pois um maior número desta resposta indica um maior nível de comportamento enxuto dos fiscais.

\section{ANÁLISE E DISCUSSÃO DOS RESULTADOS}

O questionário foi submetido entre novembro e dezembro de 2020 para respostas dos Agentes de Infraestrutura Predial de um Órgão Público Federal ligado ao Ministério da Justiça, de todos os estados da Federação. Salienta-se que, em média, há dois fiscais por estado brasileiro. Ao final, obteve-se 23 respondentes de 16 estados da Federação, mais o Distrito Federal, como mostra a Figura 1. Todas as regiões foram alcançadas, com destaque para a região Nordeste, que representou 52\% dos respondentes. Por outro lado, na região Centro-Oeste, apenas o Distrito Federal (DF) respondeu ao questionário.

Quanto à faixa etária, a idade dos fiscais varia entre 25 e 64 anos. As maiores incidências são de profissionais entre 45 e 54 anos, representando 35\% dos respondentes, seguido dos profissionais de 25 a 34 anos, representando 30\%. Quanto à formação profissional, os respondentes apresentam um perfil homogêneo que converge nas atribuições que o cargo exige. Dentre as 23 respostas, apenas 3 profissões foram citadas, sendo elas Engenharia Civil, com 15 respostas, Arquitetura e Urbanismo, com 5 respostas, e Outras Engenharias, com 2 respostas. 
Figura 1 - Distribuição dos respondentes, por estado.

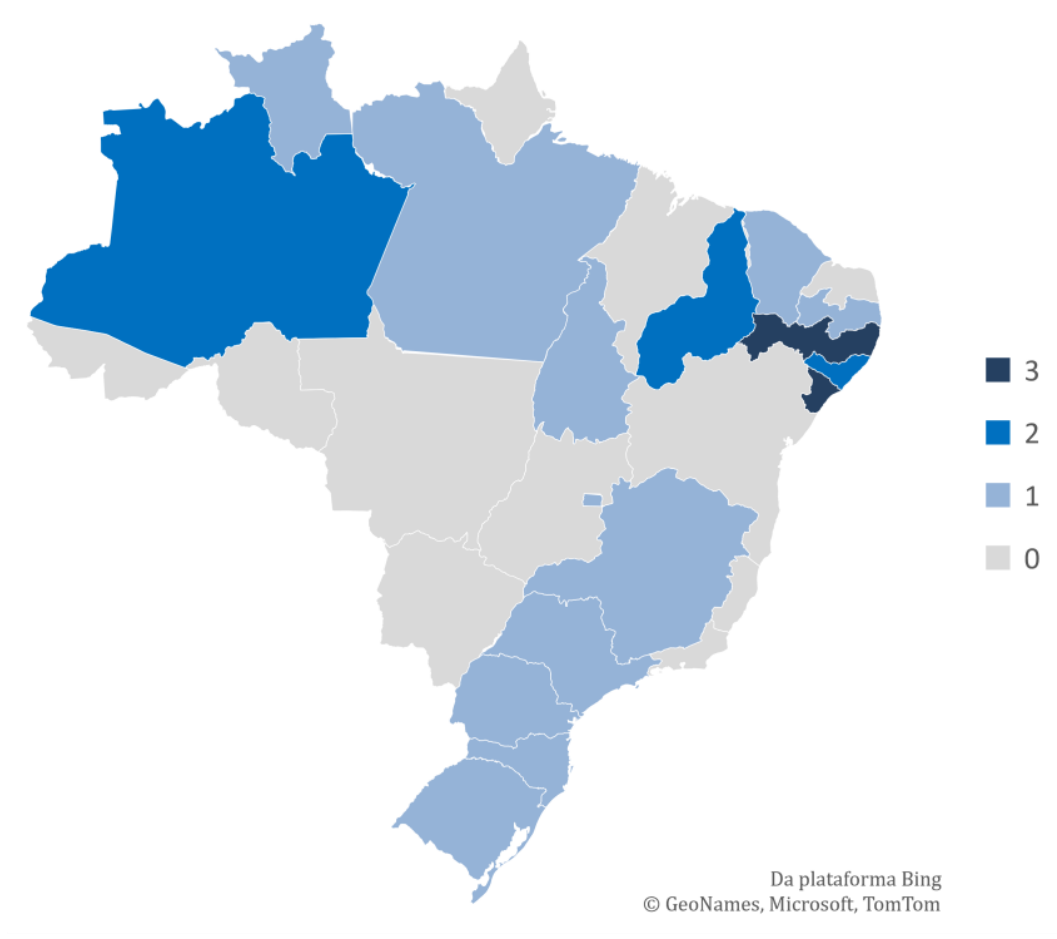

Fonte: Autores (2021)

\subsection{Desempenho de cada UF respondente de acordo aos grupos do questionário "Você tem comportamento lean?"}

A divisão do questionário em grupos de perguntas direciona para a identificação de quais boas práticas do comportamento enxuto fazem parte da rotina de cada um dos respondentes. Na Figura 2, são apresentadas as relações entre os grupos de perguntas com cada estado respondente, separados por região. Quanto mais próximo a linha estiver da extremidade do gráfico em rede, maior é o nível de conhecimento enxuto que o estado apresenta. Da mesma forma, quanto mais distante da extremidade e mais próximo do centro do radar, significa que o estado apresenta um baixo nível de conhecimento lean naquele determinado grupo.

Iniciando pela Região Norte, dos sete estados, quatro participaram do questionário. Pela análise do gráfico, destacam-se os Grupos 03, 04 e 05 mostrando que os respondentes se preocupam com a boa transmissão de informações, motivação e divisão de responsabilidades com os trabalhadores, além dos cuidados com o uso e manutenção preventiva dos equipamentos. O Grupo 01 e 02 representam as menores médias entre os estados, o que aponta um alerta por serem grupos que tratam da definição, pacotização e medição do trabalho. Entre os quatro estados, Amazonas e Tocantins apresentam os melhores resultados.

Partindo para a região Nordeste, dos nove estados, seis participaram da pesquisa. É possível observar um padrão homogêneo em relação às médias de cada estado em cada grupo. Apesar das médias bastante próximas entre os estados, poucos apresentaram médias elevadas nos grupos. Destaque no Nordeste vai apenas para os estados Ceará e Paraíba, que apresentaram melhor desempenho no Grupo 01, apresentando uma percepção boa em relação à definição e pacotização do trabalho. 
Figura 2 - Desempenho de cada UF respondente por grupo.
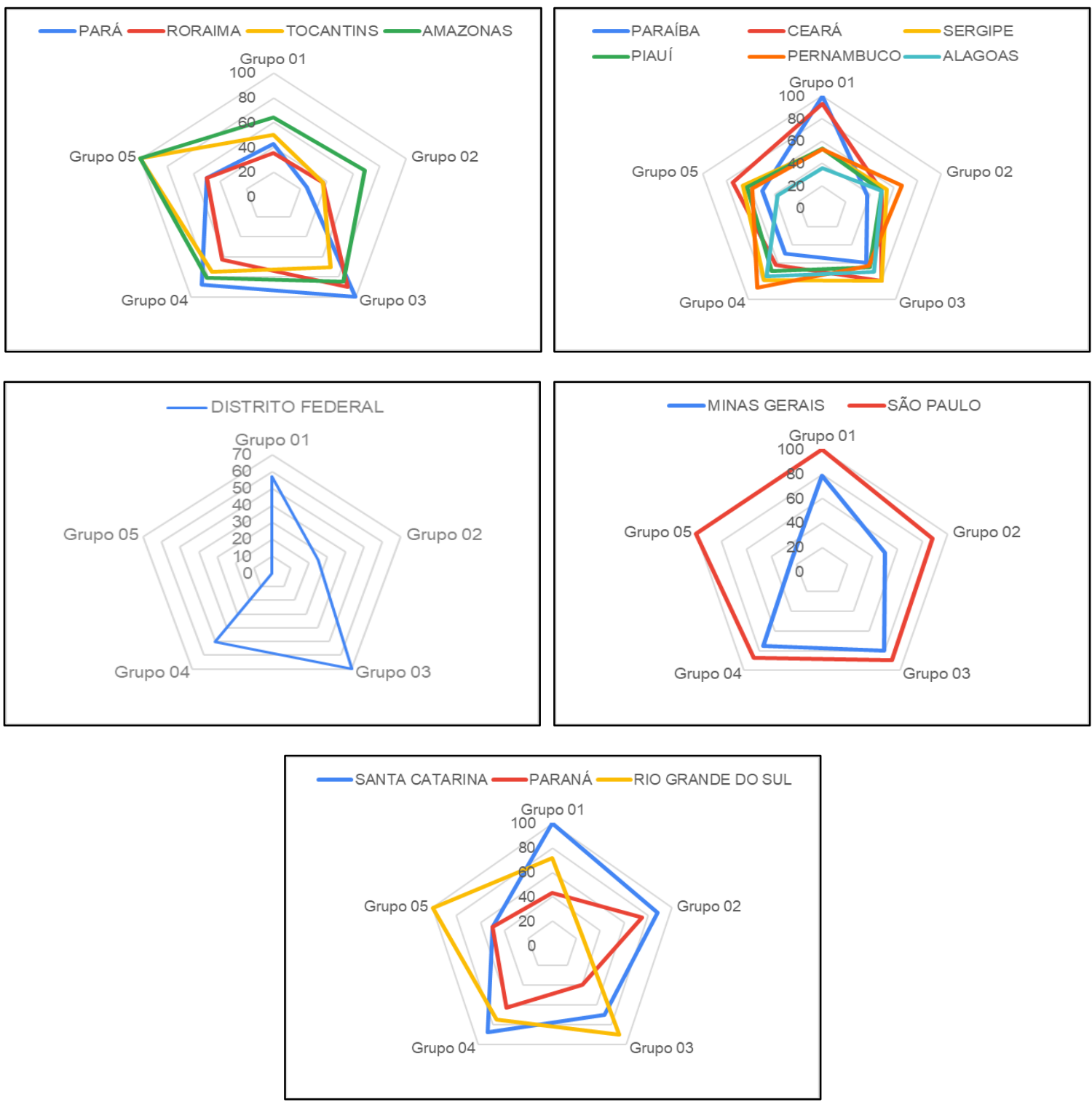

Fonte: Autores (2021)

Tratando-se da Região Centro-Oeste, a adesão ao questionário foi a menor conforme já mencionado, com apenas um participante no Distrito Federal. Neste caso, o gráfico sozinho não é suficiente para a caracterizar a região Centro-Oeste pelo baixo número de respondentes, pois a análise fica restrita a uma opinião individual. Sendo assim, é possível inferir do gráfico que os Grupos 02 e 05 representam as menores médias, caracterizando um baixo conhecimento na medição do trabalho e o uso e manutenção preventiva dos equipamentos. Já o Grupo 03 destaca-se como a melhor média entre os grupos, mostrando uma preocupação com a disseminação de informações.

Dos quatro estados da região Sudeste, houve adesão de dois. Pelo gráfico, é possível observar a excelência do conhecimento lean no estado de São Paulo, apresentando as melhores médias nos cinco grupos de respostas. Minas Gerais, o segundo estado participante, apresenta sua menor média no Grupo 05, apresentando baixo desempenho em um uso enxuto de equipamentos.

Por fim, na região Sul todos os estados participaram do questionário. Pela análise do gráfico, é possível inferir que todos os estados apresentam médias positivas em alguns grupos e médias menores em outros. Santa Catarina destaca-se obtendo a melhor média em três, dos cinco grupos do questionário, sendo eles os Grupos 01, 02 e 04, mostrando 
uma percepção boa em relação à definição e medição do trabalho e preocupação quanto à motivação e divisão de responsabilidades com os trabalhadores.

Analisando o panorama do órgão em relação aos grupos, ou seja, conhecendo o perfil do Agente de Infraestrutura Predial, têm-se o desempenho apresentado na Figura 3.

Figura 3 - Desempenho do Órgão por grupo.

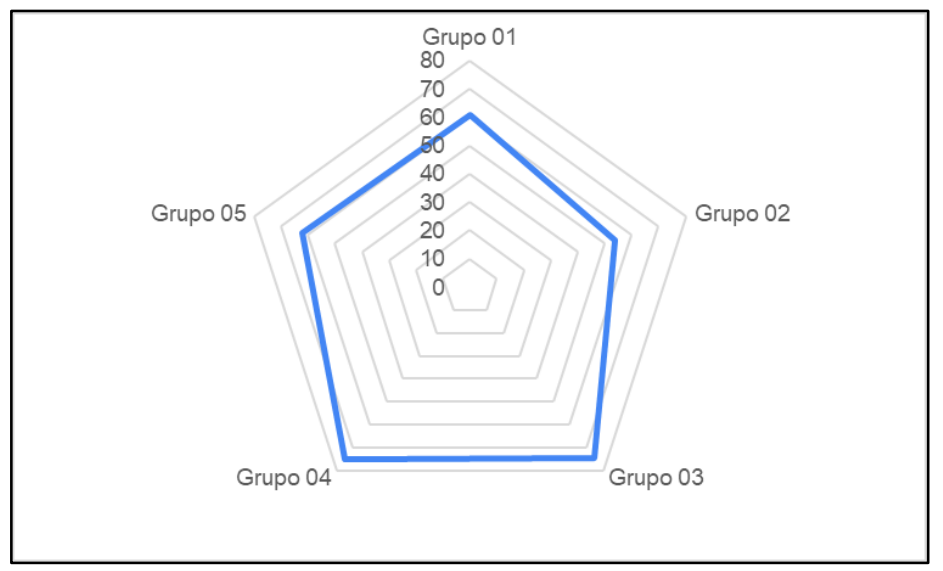

Fonte: Autores (2021)

É possível observar destaque nos grupos 03 e 04, mostrando que o órgão desenvolve habilidades dos seus membros no que se trata de comunicação, disseminação de informações e motivação dos trabalhadores. A área de maior deficiência reflete-se no grupo 02 , onde os agentes lidam com a medição do trabalho, lacuna em umas das áreas de principal atuação na função do Agente de Infraestrutura Predial. É interessante frisar que quatro, dos cinco grupos estudados, possuem índices acima de 60\%. Esse desempenho pode estar associado ao fato dos fiscais, em sua maioria, terem formação profissional compatível com as atribuições do cargo. A perspectiva global traz subsídios ao órgão para desenvolver as habilidades onde os respondentes apresentaram menor nível de conhecimento.

Comparando, agora, os resultados obtidos neste trabalho com os resultados obtidos por outros trabalhos que avaliaram o comportamento enxuto de engenheiros, observa-se que Silva et al. (2019) avaliou o comportamento de fiscais de obras públicas e também apontou um melhor domínio dos respondentes na área de comunicação, motivação e melhoria das condições de trabalho, convergindo com os resultados encontrados neste trabalho e reforçando essa característica de fiscais.

Quando os resultados desta pesquisa são comparados com os de engenheiros da iniciativa privada, apresentados em outros trabalhos, nota-se que esses últimos apresentam maior domínio quando se trata da definição do trabalho e do uso de equipamentos, indicando um comportamento oposto ao dos fiscais de obras púbicas, que mostram dificuldades nestas áreas. Por outro lado, os fiscais, quando comparados com engenheiros da iniciativa privada, apresentam melhor desempenho nos grupos que avaliam a comunicação, tópico essencial para o sucesso das atividades da Construção Civil. De forma geral, ambos os setores, público e privado, apresentam um domínio deficiente quando se trata de medição do trabalho, apontando a necessidade de maiores capacitações voltadas a este eixo, principalmente para os fiscais, em que a atividade de medição é uma das principais atribuições (SILVA et al., 2019; CRUZ e SANTOS, 2015; BRESSIANI et al., 2003).

\section{CONCLUSÃO}

De maneira geral, os cinco grupos apresentam perspectivas distintas e necessárias para construção do conhecimento lean no perfil profissional. Analisando os níveis de conhecimento por grupo e comparando-os com os de outro trabalho que avaliou o 
comportamento de fiscais, observa-se que, de maneira geral, estes possuem um melhor desempenho nos Grupos 03 e 04 e possuem menos conhecimento acerca dos demais grupos.

Este comportamento mostra um maior domínio nas áreas de comunicação, motivação e melhoria das condições de trabalho, ao passo que apresenta o menor domínio quando se trata de valor, ciclo, fluxo, desperdícios, padronização, tecnologia básica, medição e melhoria da produção, pontos fortes dos profissionais atuantes no setor privado. Em contrapartida, quando se trata de comunicação, os fiscais de obras públicas possuem melhor desempenho frente aos profissionais do setor privado que foram avaliados em outros trabalhos.

De maneira geral, os fiscais dominam melhor questões que são do nível operacional, apresentando menor conhecimento em questões de nível estratégico. É sabido que a aplicação de conceitos de gestão tidos como modernos, a exemplo dos enxutos, são mais facilmente difundidos no meio operacional, sobretudo através dos canteiros de obras. Porém, a presença dos conceitos a nível estratégico é essencial ao sucesso da implementação nas organizações, contribuindo na maturidade destas.

Posto isso, é importante ressaltar que as informações trazem para a Administração Pública uma visão ampla da disseminação de conhecimento lean por Unidade Federativa, pontuando as deficiências que necessitam de maior atenção e fornecendo base para tomadas de decisão acerca de investimentos em princípios modernos de gestão, como a Construção Enxuta. Intensificar a capacitação nas áreas de menor domínio tendem a aumentar a efetividade da aplicação dos conceitos, trazendo ganhos mais expressivos à Administração. Isso contribui para a tomada de decisão não apenas desse órgão público pesquisado, mas de outros com abrangência de trabalho de modo nacional.

Para suprir as lacunas de conhecimento, por parte dos fiscais, sugere-se parcerias entre os órgãos públicos e a comunidade acadêmica, visando capacitação e consolidação da Construção Enxuta e outras filosofias modernas de gestão.

Em pesquisas futuras, sugere-se a aplicação do questionário associada a uma verificação de resultados obtidos nas organizações por meio da aplicação de conceitos enxutos. Outro caminho que pode ser explorado, é o desenvolvimento de estratégias para disseminar os conceitos enxutos em ambientes que pouco os dominam.

\section{REFERÊNCIAS}

ALVARENGA, F. C. Análise das causas de aditivos de custo e de prazo em obras públicas de instituições federais de ensino. 2019. 114p. Dissertação (Mestrado em Engenharia Civil) - Programa de Pós-Graduação em Engenharia Civil. Universidade Federal do Pará, Belém, 2019.

ANDÚJAR-MONTOYA, M. D.; GALIANO-GARRIGÓS， A.; ECHARRIIRIBARREN, V.; RIZO-MAESTRE C. BIM-LEAN as a Methodology to Save Execution Costs in Building Construction-An Experience under the Spanish Framework. Applied Sciences. v. 10, n. 6, p.1913, 2020.

BABALOLA, O.; IBEM, E. O.; EZEMA, I. C. Implementation of lean practices in the construction industry: A systematic review. Building and Environment, v. 148, p. 3443, Jan. 2019.

BRANDSTETTER, M. C. G. de O.; RIBEIRO, H. R. de O. Causas de custos adicionais e impacto financeiro em obras públicas sob a perspectiva da gestão de risco. Ambiente Construído, Porto Alegre, v. 20, n. 1, p. 41-63, Mar. 2020. Epub 05 Dez., 2019. 
BRESSIANI, L.; SANTOS, D. G.; SAFFARO, F. A.; SOUZA e SILVA, M. F.; HEINECK, L. F. O. O comportamento dos engenheiros de obra em relação aos princípios lean. In: SIMPÓSIO BRASILEIRO DE GESTÃO E ECONOMIA DA CONSTRUÇÃO, 2003, São Carlos. Anais... São Carlos, 2003.

CASOTTE, A. G. Z. Gestão de obras públicas: análise dos aditivos financeiros e temporais nos contratos de obras da Universidade Federal do Espírito Santo no período de 2009 a 2015. 2016. 127 f. Dissertação (Mestrado Profissional em Gestão Pública) Universidade Federal do Espírito Santo. Vitória. 2016.

CORRÊA, B. dos S.; SHIH, H. H. O. M. Gestão da obra pública: uma análise comparativa dos aditivos de valor e de prazo entre duas instituições federais de ensino. Revista Gestão Universitária na América Latina - GUAL, Florianópolis, v.12, n. 3, p. 130-150, set. 2019. ISSN 1983-4535.

CRUZ, H. M.; SANTOS, D. G. Investigação do comportamento enxuto de empresas construtoras de médio porte. In: SIMPÓSIO BRASILEIRO DE GESTÃO E ECONOMIA DA CONSTRUÇÃO, 2015, São Carlos. Anais... São Carlos, 2015.

HEINECK, L. F. H.; et al. Coletânea Edificar Lean: Construindo com Lean Management, v.1. Fortaleza: Expressão gráfica Editora, 2009.

IBGE. Instituto Brasileiro de Geografia e Estatística. Pesquisa Anual da Indústria da Construção - PAIC: Tabelas 2018. Disponível em: https://www.ibge.gov.br/estatisticas/economicas/industria/9018-pesquisa-anual-daindustria-da-construcao.html?=\&t=resultados. Acesso em julho de 2020.

SILVA, J. L. O.; CRUZ, H. M.; SANTOS, D. G. Comparação entre o comportamento enxuto de engenheiros do setor público e do setor privado. In: SIMPÓSIO BRASILEIRO DE GESTÃO E ECONOMIA DA CONSTRUÇÃO, 11., 2019, Londrina. Anais [...]. Porto Alegre: ANTAC, 2019.

WEBER, L. E. Aditivos de prazo em obras públicas: uma análise sob a ótica dos empreiteiros e a aplicabilidade dos conceitos enxutos. 2020. 96 f. Dissertação (Mestrado em Engenharia Civil) - Programa de Pós-Graduação em Engenharia Civil, Universidade Federal de Sergipe, São Cristóvão, 2020.

\section{AGRADECIMENTOS}

Ao órgão objeto de estudo, pela disponibilidade de realização da pesquisa. 